\title{
Medikamentenverordnung: einen komplexen Prozess sicherer machen
}

- Die Verordnung von Medikamenten ist eine komplexe und fehleranfällige ärztliche Tätigkeit. Es ist wichtig, dass das Bewusstsein dafür in der Ärzteschaft noch mehr verankert wird.

- Die Ursachen von Verordnungsfehlern sind häufig systembedingt. Die FMH erachtet daher interdisziplinäre, institutionsübergreifende und multidimensionale Verbesserungsansätze als wichtig. Zudem sind vor allem für den ambulanten Bereich weitere Ursachenanalysen notwendig.

- Viele Verordnungsfehler sind vermeidbar. Es gibt aber bereits praxistaugliche Instrumente, welche die Sicherheit bei der Medikamentenverordnung erhöhen. Man sollte sie daher breiter einsetzen und koordinieren.

- Ein offener und konstruktiver Umgang mit Verordnungsfehlern soll gefördert werden, denn er bildet das Fundament für konkrete Verbesserungsmassnahmen.

Korrespondenz: FMH/Abteilung DDQ Elfenstrasse 18 CH-3000 Bern 15 Tel. 0313591111 Fax 0313591112 ddq[at]fmh.ch

\section{Die Ausgangslage}

Sowohl im ambulanten als auch im stationären Sektor gilt die Medikationssicherheit als einer der wichtigsten Hot Spots der Patientensicherheit. So sieht auch die Qualitätsstrategie des Bundes im Schweizerischen Gesundheitswesen einen Schwerpunkt bei der Verbesserung der Medikationssicherheit, gleich wie verschiedene andere Länder und Organisationen, beispielsweise die WHO. Die ärztliche Medikamentenverordnung nimmt einen zentralen Stellenwert im gesamten Medikationsprozess ein - einem wichtigen, wenn auch sehr komplexen und besonders fehleranfälligen Prozess. International wie auch in der Schweiz existieren erprobte und vielversprechende Ansätze, um die Verschreibung von Medikamenten sicherer zu machen.

\section{Die Position der FMH}

Häufigkeit von Verordnungsfehlern: Nationale und internationale Studien zeigen, dass Verordnungsfehler häufig sind, obwohl sich viele davon vermeiden liessen. An erster Stelle leiden die Patienten darunter. Aber auch die finanziellen Folgen sind von Bedeutung. Die FMH ist klar der Meinung, dass Verordnungsfehler deutlich zu häufig auftreten. Neben konkreten Fehlerquellen wie beispielsweise Missverständnissen gilt vor allem das fehlende Bewusstsein für die hohe Fehleranfälligkeit der Medikamentenverordnung als Ursache für Verordnungsfehler. Um dieses Bewusstsein in der Ärzteschaft zu fördern sollte die Problematik in der ärztlichen Aus- und Weiterbildung noch stärker verankert werden. Erst die dadurch erzeugte Wachsamkeit ermöglicht es,
Verschreibungsprozesse präzise zu überprüfen und zu optimieren.

Ursachen von Verordnungsfehlern: Die Verordnung von Medikamenten ist ein komplexer Prozess. Ebenso vielschichtig stellt sich auch die Ursachenanalyse von Verordnungsfehlern dar. Häufig führen nicht einzelne Faktoren zu Fehlern, sondern eine Verknüpfung von fehlerbegünstigenden Umständen. Vor allem im ambulanten Bereich sind die Ursachen bisher jedoch noch ungenügend erforscht. Weitere Studien sind notwendig, damit sich die Verbesserung der Verordnungssicherheit datenbasiert möglichst spezifisch angehen lässt.

Umgang mit Verordnungsfehlern. Zentral für eine systematische Analyse von Verordnungsfehlern sind ein offener Umgang mit diesen - beispielsweise auf der Basis eines Fehlermeldesystems - sowie eine konstruktive Fehlerkultur. Dadurch lässt sich verhindern, dass vermeidbare Verordnungsfehler immer wieder auftreten. Da Verordnungsfehler häufig eine systembedingte Ursache haben, sind dazu auch berufsgruppenübergreifende Analysen und Diskussionen z. B. im Rahmen von Qualitätszirkeln wichtig.

Verbesserung der Verordnungssicherheit: Da Medikationsfehler reduziert werden müssen, ist die Verordnungssicherheit $\mathrm{zu}$ optimieren. Es existieren bereits zahlreiche erprobte und vielversprechende Instrumente für die Prävention von Verordnungsfehlern wie beispielsweise elektronische Verordnungssysteme oder berufsübergreifende Qualitätszirkel zwischen Ärzten und Apothekern. Diese sollten unterstützt und koordiniert werden, damit sich aus bewährten Einzelansätzen grossflächigere Massnahmen realisieren und verankern lassen. Weiter sollten auch neue praxistaugliche Instrumente entwickelt und umgesetzt werden. Die FMH-AG eHealth hat spezifische Grundlagen und Positionen hinsichtlich der elektronischen Unterstützung der Verordnung erarbeitet und wird diese in Kürze publizieren.

Monitoring: Um die Verordnungssicherheit nachhaltig zu verbessern, sind wirksame Massnahmen notwendig. Um zu kontrollieren, ob die ergriffenen Schritte die Verordnung von Medikamenten wirklich verbessern, ist ein regelmässiges Monitoring sinnvoll. Damit sind Studien gemeint, die längerfristig das Vorkommen von Verordnungsfehlern erheben sowie die Wirkung konkret umgesetzter Verbesserungsmassnahmen überprüfen. 